\title{
Application of Computer 3D Simulation in Architectural Art Effect
}

\author{
Wei Peng \\ Architecture and Art Branch, Yiwu Industrial and Commercial College, Yiwu, China
}

Keywords: BIM technology, Architectural art, REVIT software, 3D modeling, Simulation

\begin{abstract}
Computer 3D simulation technology increases three-dimensional and visualization of architectural art form, which has the vital significance to explore the design technology of modern architectural art. This paper uses BIM and REVIT software to build the integrated platform of architecture art, and the functions of the platform include 3ds Max 3D modeling design and the artistic rendering effect of Photoshop. Through the 3D modeling and color tone rendering, the building will be eventually shown in 3D visualization form, achieving the perfect combination of art and design.
\end{abstract}

\section{Introduction}

The art of architecture refers to the law of beauty, and uses the unique architectural art language, so the building image has cultural value and aesthetic value, with symbolic and formal beauty, reflecting the national character and sense of the times. This paper takes the BIM building information modeling as integrated platform, through the Revit data interface, and uses 3ds max software to design the art shape of building structure [1,2]. Finally it uses Photoshop software to do rendering on the architecture structure, and the 3D art expression effect is obtained, which gives full play to the functions of the computer virtual design and provides the theoretical basis for building aesthetic design.

\section{The Virtual Framework Design of BIM Architecture Art}

The 3ds Max has been widely used in advertising, film and television, industrial design, architecture design, 3D animation, multimedia, games and engineering visualization fields, which is powerful 3D modeling software. Photoshop software can effectively edit the photo, increasing 3D image artistic effect [3]. Combining with the two soft wares, this paper designs the platform framework of architectural art effect as shown in Figure 1.

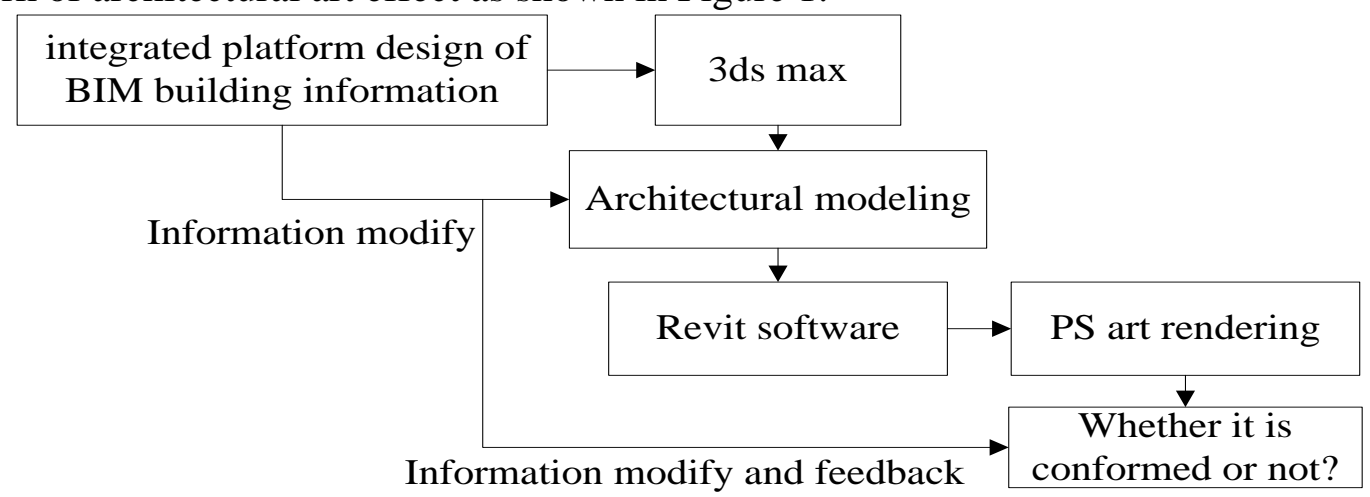

Fig. 1 The architectural art design platform

Figure 1 shows the schematic design of the architectural art effect. As shown in the figure, this paper adopts the BIM architecture information integration platform to develop the structure model and the artistic effect of building, and uses 3ds max to design the 3D architectural model, through the Revit software realizing the integrated data interface [4]. It uses Photoshop to do visual rendering on the construction color art effect through the data interface, finally through the feedback of information it modified and optimized the building structure, which greatly improves the design efficiency of the building. 


\section{BIM Virtual Mathematics Model and Algorithm Design based on Finite Element Method}

Using BIM virtual technology can realize the architecture integrated development, and 3D modeling can optimize the design process, which is based on the finite element theory [5-7]. The whole building rendering artistic effect needs to render local each unit body, which can render from the three direction of 3D solid element respectively, and the formula is as follows:

$$
\left\{\begin{array}{l}
d G x(a, b, c)=J x 1 l(a, b, c) d r+J x 2 \frac{d r}{\cos \theta} \\
d G y(a, b, c)=J y 1 l(a, b, c) d r+J y 2 \frac{d r}{\cos \theta} \\
d G z(a, b, c)=J z 1 l(a, b, c) d r+J z 2 \frac{d r}{\cos \theta}
\end{array} .\right.
$$

Where, $d G x(a, b, c), d G y(a, b, c)$ and $d G z(a, b, c)$ are artistic effect render functions in three directions; $J x 1$ is longitudinal rendering coefficients in three directions; $J x 2$ is the transverse rendering coefficients in the three directions; $l(a, b, c)$ is rendering thickness; $l(a, b, c) d r$ is rendering area.

$$
l(a, b, c) d r=\left\{\begin{array}{c}
\operatorname{fr} \cos (\beta(a, b, c)) \\
0
\end{array} \quad \beta(a, b, c) \in[\psi 1, \psi 2] .\right.
$$

Through coordinate transform method, the finite element coordinates are changed to 3D model, and the transformation matrix can be expressed as:

$$
\left[\begin{array}{l}
d G s(a, b, c) \\
d G d(a, b, c) \\
d G w(a, b, c)
\end{array}\right]=\left[\begin{array}{l}
d G x(a, b, c) \\
d G y(a, b, c) \\
d G z(a, b, c)
\end{array}\right] \cdot T \text {. }
$$

After put into $T$, finite element model of the whole 3D architectural art rendering can be expressed as:

$$
\left\{\begin{array}{l}
G x(b)=\sum_{i=1}^{M} \sum_{u=1}^{N} d G x(a, b, c) \\
G y(b)=\sum_{i=1}^{M} \sum_{u=1}^{N} d G y(a, b, c) . \\
G z(b)=\sum_{i=1}^{M} \sum_{u=1}^{N} d G z(a, b, c)
\end{array}\right.
$$

Where, $G x(b), G y(b)$ and $G z(b)$ are total rendering effect in three axial, while $N$ indicates the finite element number of involved in rendering.

\section{D Simulation of Architectural Art Effect}

In order to verify the effectively and reliability of 3D building the finite element virtual computing mathematical model and algorithm designed in second part, this paper uses the Revit Architecture of BIM to integrate the architectural art effect, including the 3ds max modeling and Photoshop rendering process [8,9]. Eventually buildings 3D visualization form is showed, reaching the perfect combination of art and design. The BIM integrated environment is as shown in Figure 2. 


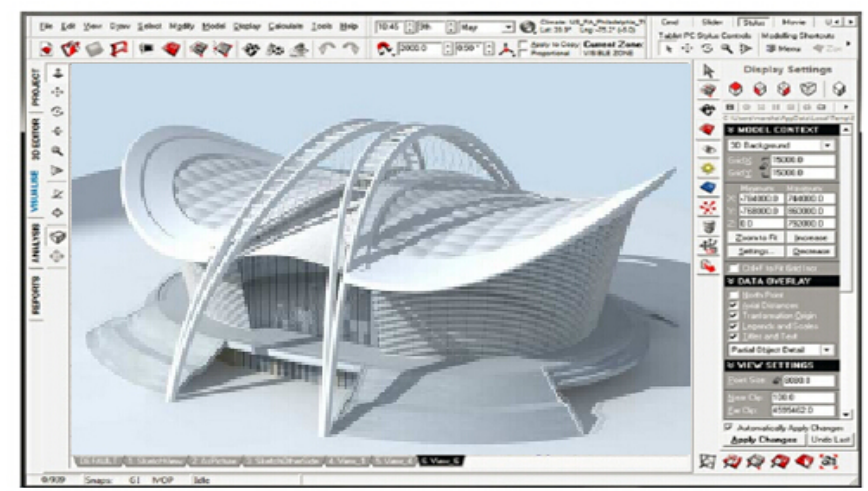

Fig. 2 The architectural art integration design

Autodesk Revit Architecture software is the special software of building construction information model, which can realize the integrated design of modern artistic effect based on building information model. The 3D model design is as shown in Figure 3:

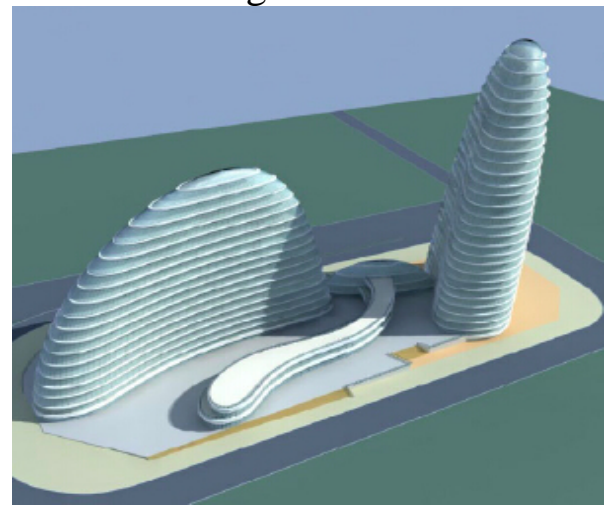

Fig. 3 3ds max software modeling

Figure 3 shows the building entity model using $3 \mathrm{ds}$ max software. The version of the $3 \mathrm{ds}$ max in this paper is 2013. This version updated powerful polygon drawing function, which greatly improves the construction art design effect [10-12]. Figure 3 shows 3D construction using curves and surfaces, and its overall shape has good artistic expression effect.

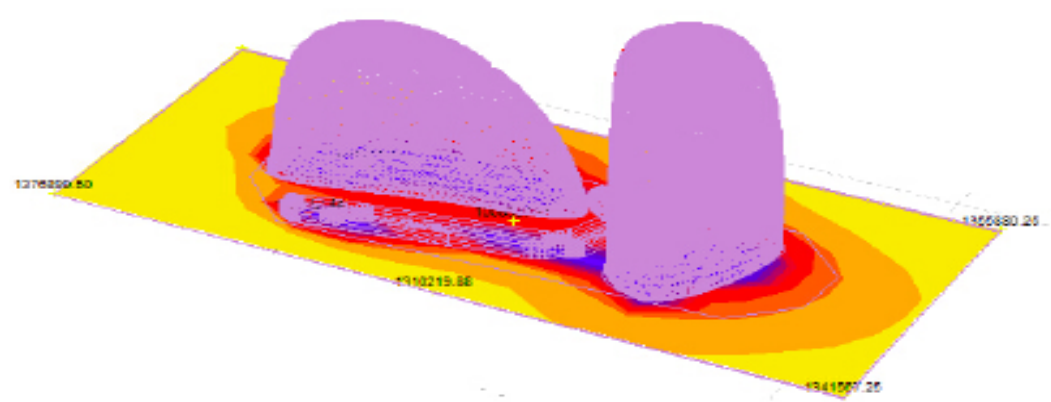

Fig. 4 Rendering process of building structure artistic

Figure 4 shows the art rendering process of building structures. In order to enhance the construction artistic effect, this paper uses Photoshop to do further rendering on architectural image, by adding different colors to highlight the building art effect.

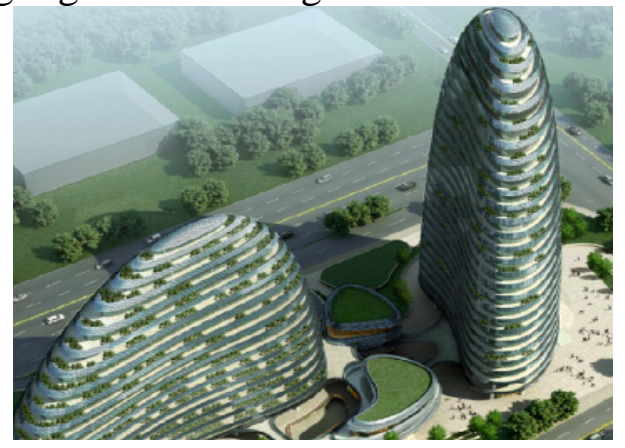

Fig. 5 The construction ecological art renderings 
By modeling and artistic rendering of architecture structure, finally the ecological architecture art effect is obtained as shown in Figure 5. It uses 3ds max to establish the external decoration of flowers and trees, and uses Photoshop to obtain the basic color art effect of green, successfully realizing computer visualization of architectural art.

\section{Summary}

Combining with the finite element theory, this paper establishes the mathematical model of architecture art effect rendering, with BIM building information modeling as integrated platform, through the Revit data interface, develops the architecture art design system. Through the system testing, the system can create various multilateral arts building structure, and can render different colors, achieving a perfect combination of architectural design, art and computer, which provides a theoretical reference for the study on architecture aesthetics design.

\section{References}

[1] D.H. Li, R.Z. Zhang. Application of BIM technology in 3D modeling of digital city. Information technology in building and civil engineering, 2012, 4(3): 47-51.

[2] G.P. He, G. Li. BIM will bring what changes to the building industry. Information, 2011, 3(2): 16-20.

[3] J. Ren, X.B. Wang. Study on BIM theory in construction project life cycle. The third theory for engineering construction computer application, 2011, 4(3): 231-237.

[4] Z.K. Li. Application of BIM in building sustainable design. Journal of Suzhou University of Science and Technology, 2012, 4(6): 68-71.

[5] G.C. Liang. Explore on the city office building architecture design. Information technology, 2012, 2(3): 72-75.

[6] Z.Q. Liu, Y.G. Li. Development of building information model and its application in the design. Building science, 2012, 25(1): 101-104.

[7] Y. Chen. Cable membrane structure CAD development based on ObjectARX. South China University of Technology, 2012, 5(2): 45-48.

[8] BIM application report of China commercial real estate. Commercial real estate professional committee China Real Estate Association, 2012.

[9] W.P. Shao, Y.G. Liu, L.C. Xiao. The Phoenix Nirvana - digital design platform builds the Phoenix Painting media centre. Journal of architecture, 2012, 3(1): 87-89.

[10]Y.B. Cheng. Application of the SketchUp software in the conception of architectural design. Harbin Institute of Technology, 2012: 2-16.

[11]Y. Long, X. Li. The building exterior wall thermal insulation energy-saving technology route in Chongqing area. Building energy saving, 2011, 5(6): 21-24.

[12]X.F. Zhang, Y.C. Zhou. BIM technology application field and its prospect analysis based on IFC standard. Building science, 2012, 4(10): 94-97. 